\title{
Science Opportunities with the Near-IR Camera (NIRCam) on the James Webb Space Telescope (JWST)
}

Charles A. Beichman ${ }^{* a, b}$, Marcia Rieke ${ }^{c}$, Daniel Eisenstein ${ }^{\mathrm{d}}$, Thomas P. Greene ${ }^{\mathrm{e}}$, John Krist ${ }^{\mathrm{b}}$

Don McCarthyc, Michael Meyer ${ }^{\mathrm{f}}$, John Stansberry ${ }^{\mathrm{b}}$, and the NIRCam Team

aNASA Exoplanet Science Institute, IPAC 100-22, Pasadena, CA 91125; ' Jet Propulsion Laboratory,

California Inst. of Technology; Pasadena, CA 91125; 'Steward Observatory, Univ. of Arizona, Tucson, AZ; ; ${ }^{\mathrm{d}}$ Harvard University, Cambridge, MA 01238; ${ }^{\mathrm{N}} \mathrm{NSA}$ Ames Research Center, Moffett

Field, CA 94035; ${ }^{\mathrm{E}}$ ETH Zurich, Institute for Astronomy, 8093 Zurich, Switzerland.

\begin{abstract}
The Near-Infrared Camera (NIRCam) on the James Webb Space Telescope (JWST) offers revolutionary gains in sensitivity throughout the 1-5 $\mu \mathrm{m}$ region. NIRCam will enable great advances in all areas of astrophysics, from the composition of objects in our own Kuiper Belt and the physical properties of planets orbiting nearby stars to the formation of stars and the detection of the youngest galaxies in the Universe. NIRCam also plays an important role in initial alignment of JWST and the long term maintenance of its image quality. NIRCam is presently undergoing instrument Integration and Test in preparation for delivery to the JWST project. Key near-term milestones include the completion of cryogenic testing of the entire instrument; demonstration of scientific and wavefront sensing performance requirements; testing of replacement H2RG detectors arrays; and an analysis of coronagraphic performance in light of
\end{abstract}

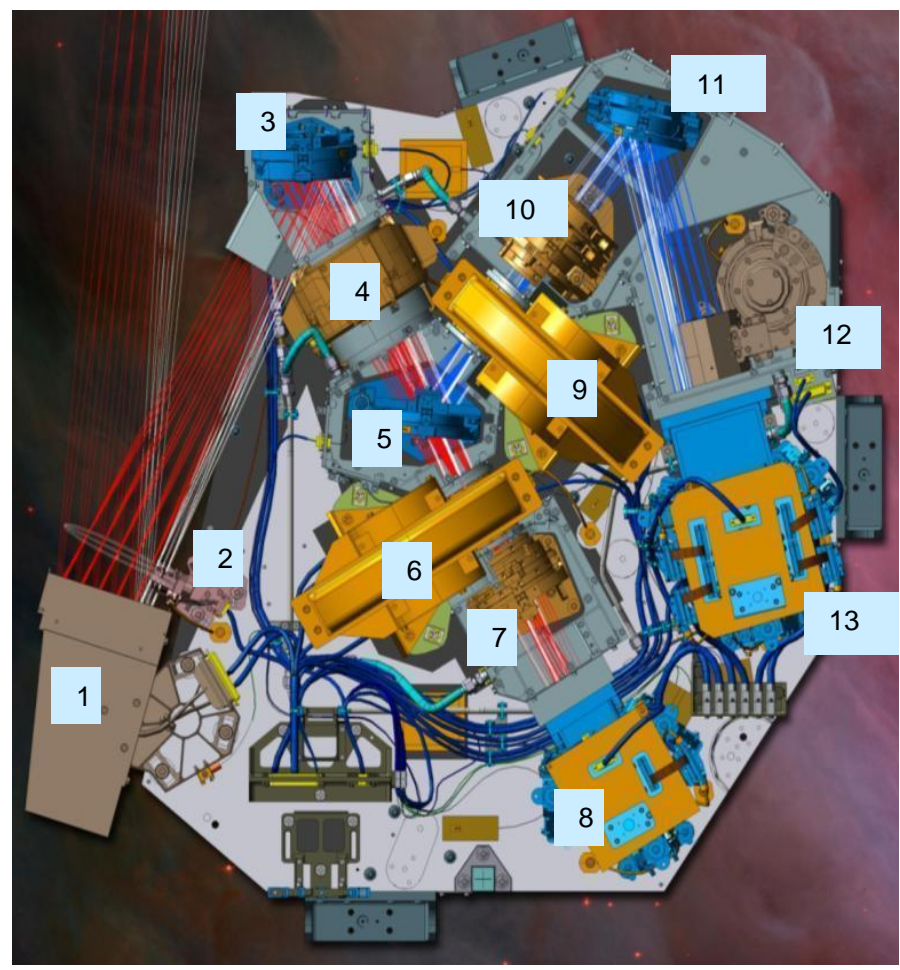

\begin{tabular}{r|l|}
\hline 1 & Pick-off Mirror assembly \\
\hline 2 & Coronagraph \\
\hline 3 & First Fold Mirror \\
\hline 4 & Collimator lens group \\
\hline 5 & Dichroic Beamsplitter \\
\hline 6 & Longwave Filter Wheel Assembly \\
\hline 7 & Longwave Camera lens group \\
\hline 8 & Longwave Focal Plane \\
\hline 9 & Shortwave Filter Wheel Assembly \\
\hline 10 & Shortwave Camera lens group \\
\hline 11 & Shortwave Fold Mirror \\
\hline 12 & Pupil Imaging Lens \\
\hline 13 & Shortwave Focal Plane \\
\hline & \\
\hline
\end{tabular}

Figure 1 The short and long wavelength channels of NIRCam share a common optical bench. An identical module ("B") is bolted to the backside of module A.

\footnotetext{
"chas@ipac.caltech.edu; phone 1818-653-8220; fax 1626-397-7018
}

Space Telescopes and Instrumentation 2012: Optical, Infrared, and Millimeter Wave, edited by Mark C. Clampin, Giovanni G. Fazio, Howard A. MacEwen, Jacobus M. Oschmann, Jr., Proc. of SPIE Vol. 8442, 84422N

(C) 2012 SPIE · CCC code: $0277-786 / 12 / \$ 18 \cdot$ doi: $10.1117 / 12.925447$ 
measured telescope wavefront characteristics. This paper summarizes the performance of NIRCam, the scientific and education/outreach goals of the science team, and some results of the on-going testing program.

Keywords: Infrared, James Webb Space Telescope, planets, solar system, star formation, galaxies

\section{INTRODUCTION}

NIRCam plays a critical role in all of JWST's scientific goals as well as providing critical capabilities in the initial acquisition of the JWST's segmented telescope and its long term wavefront control. This paper focuses on the scientific aspects of NIRCam but will touch on its facility level capabilities and responsibilities. The layout of NIRCam is depicted in Figure 1. NIRCam is a highly versatile imager ${ }^{1}$ covering the 0.6 to $5.0 \mu \mathrm{m}$ spectral range with 29 broad, medium, and narrow-band filters (Figure 2). Only a few of these filters work in the visible where JWST's advantages relative to large ground-based telescopes will be small. Configured with two identical, redundant modules each equipped with short and long wavelength cameras, NIRCam covers two 2.2'×2.2' Fields of View (FOV) --- each with more than twice the FOV of WFC3/IR on Hubble Space Telescope (HST). Depending on wavelength, NIRCam's angular resolution ranges from almost three times better than Hubble's WFC3/IR at $1.6 \mu \mathrm{m}$ to comparable resolution between HST at $1.6 \mu \mathrm{m}$ and NIRCam at $4.8 \mu \mathrm{m}$. NIRCam's images are Nyquist sampled at 2 and $4 \mu \mathrm{m}$ with 0.0317 " pixels in the short wavelength arm and 0.0647" pixels in the long wavelength arm compared with WFC3/IR's 0.13" pixels. A full description of NIRCam's basic features is available at http://ircamera.as.arizona.edu/nircam/features.html.

NIRCam's pupil wheels accommodate Lyot stops for the long and short wavelength coronagraphs ${ }^{2}$, long wavelength grisms (2.4-5 $\mu \mathrm{m}, \mathrm{R} 2000$ ) which are used both for on-orbit mirror alignment and science investigations ${ }^{3}$, and defocusing lenses intended for wavefront retrieval but which also can be used for spreading out the light from very bright stars to enable accurate transit photometry.

Ground-based sky backgrounds in the 1-2.4 $\mu \mathrm{m}$ range are $10^{2}-10^{3}$ greater than from space, giving JWST a significant sensitivity advantage relative to 8-10 m telescopes. This advantage becomes particularly striking when JWST's highly stable, near-diffraction-limited performance over a wide FOV is compared with the modest Strehl ratios and narrow FOVs possible from the ground. JWST offers a 3-4 magnitude improvement compared with Keck's NIRC2 camera

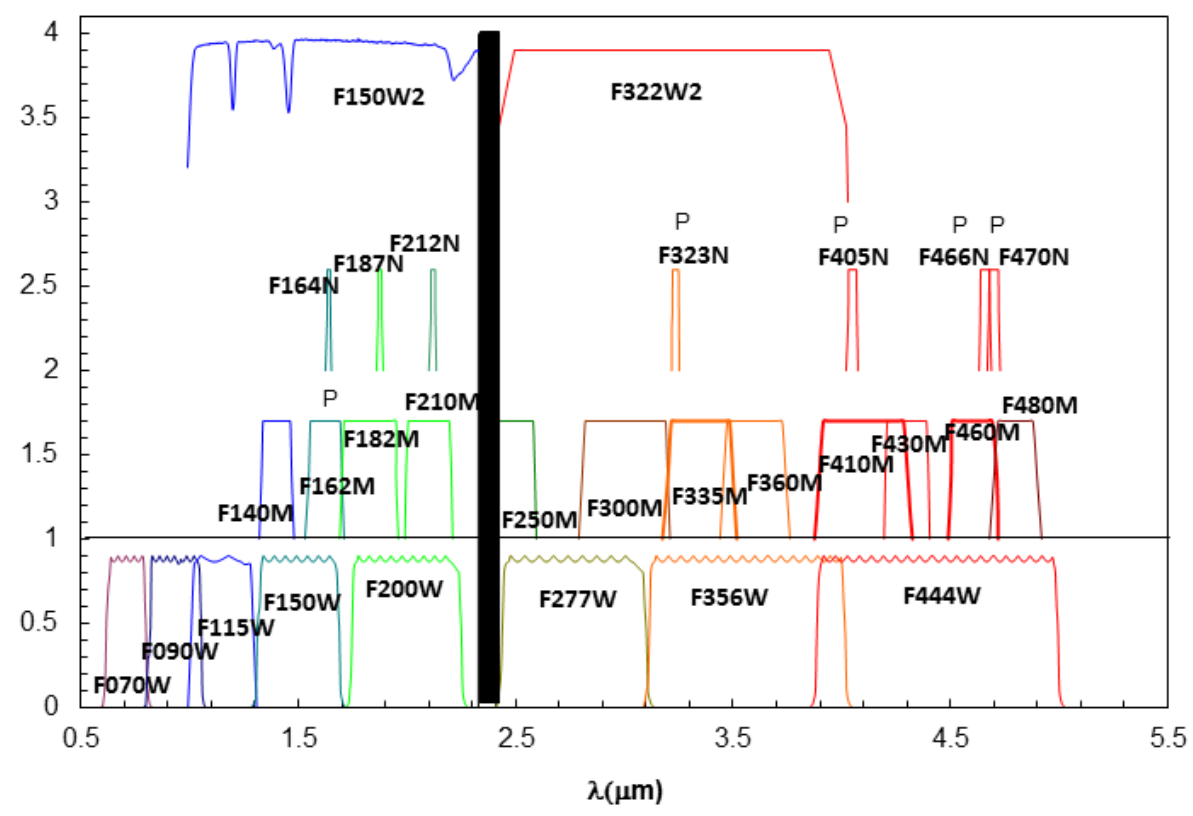

Figure 2. Graphical depiction of NIRCam filters. The vertical bar indicates the beamsplitter deadband while $\mathrm{P}$ indicates those filters in the pupil wheels. 
Table 1. Sensitivity comparison between JWST/NIRCAM, HST/WFC3, Spitzer/IRAC and the NIRC2 Instrument on the $10 \mathrm{~m}$ Keck Telescope. Estimates of the $5 \sigma$ sensitivity in $3600 \mathrm{sec}$ of integration time come from respective time estimator tools.

\begin{tabular}{|c|c|c|c|c|c|}
\hline $\begin{array}{l}\text { JWST/ } \\
\text { NIRCAM }\end{array}$ & $\begin{array}{l}\text { Point Source } \\
\text { Limit (Vega } \\
\text { mag, } 5 \sigma, 1 \mathrm{hr})^{1}\end{array}$ & $\begin{array}{l}\text { Keck NIRC-2 } \\
\text { with Natural } \\
\text { Guide Star AO }\end{array}$ & $\begin{array}{l}\text { Point Source } \\
\text { Limit (Vega } \\
\text { mag, } 5 \sigma, 1 \mathrm{hr})^{2}\end{array}$ & $\begin{array}{l}\text { HST } \\
\text { WFC3/IR or } \\
\text { Spitzer/IRAC }\end{array}$ & $\begin{array}{l}\text { Point Source } \\
\text { Limit (Vega } \\
\text { mag, } 5 \sigma, 1 \mathrm{hr})^{3,4}\end{array}$ \\
\hline F115W & 27.5 & $\mathrm{~J}$ (Strehl $=0.2)$ & 24.4 & F110W & 26.5 \\
\hline F150W & 27.5 & $\mathrm{H}($ Strehl $=0.3)$ & 23.9 & F160W & 25.5 \\
\hline F200W & 27.3 & Ks $($ Strehl=0.5) & 23.7 & & \\
\hline F300W & 26.1 & L' (Strehl=0.6) & 18.1 & IRAC $3.6 \mu \mathrm{m}$ & 21.2 \\
\hline F444W & 25.1 & M (Strehl=0.6) & 15.6 & IRAC $4.5 \mu \mathrm{m}$ & 20.4 \\
\hline
\end{tabular}

'http://jwstetc.stsci.edu/etc/input/nircam/imaging/

${ }^{2}$ http://www2.keck.hawaii.edu/cgi-bin/ion-p?page=nirc2_snr_eff.ion ${ }^{3}$ http://etc.stsci.edu/etc/input/wfc3ir/imaging/

${ }^{4}$ http://ssc.spitzer.caltech.edu/warmmission/propkit/pet/senspet/index.html

operating with Natural Guide Star Adaptive Optics (Table 1). In the 3-5 $\mu \mathrm{m}$ range where atmosphere and telescope backgrounds are $10^{4}$ to $10^{5}$ larger than in space, NIRCam's sensitivity advantages become overwhelming, between 8 to 10 magnitudes compared with Keck or other large ground-based telescopes. As will be discussed in the section on exoplanets (§2.3) it is only in the area of high angular resolution, high contrast imaging that ground-based telescopes equipped with Extreme Adaptive Optics retain a sensitivity advantage due to their superior diffraction limit and starlight rejection. Table 1 also compares NIRCam's performance with WFC3/IR on HST in the 1-1.6 $\mu \mathrm{m}$ region where JWST's larger aperture yields a significant 1-2 magnitude advantage and with Spitzer/IRAC at 3.6 and $4.5 \mu \mathrm{m}$ where the JWST's larger aperture yields a dramatic 5 magnitude gain. For deep exposures at 3-5 $\mu \mathrm{m}$ IRAC becomes confusion limited below a few $\mu \mathrm{Jy}$. JWST's $\sim 7.5$-fold larger diameter completely removes this restriction with surveys planned to go a thousand times deeper, reaching flux density levels of a few nJy (§2.1).

NIRCam's field of view, sensitivity, filter suite and other capabilities have been designed to address JWST's four overarching science goals ${ }^{4}$ :

1. Exploring the formation and evolution of the first luminous objects and revealing the reionization history of the Universe.

2. Determining how objects seen in the present day (galaxies, active galaxies, and clusters of galaxies) assembled and evolved out of gas, stars, metals present in the early Universe.

3. Improve our understanding of the birth of stars and planetary systems.

4. Study the physical and chemical conditions of objects in our solar system with a goal of understanding the origin of the building blocks of life on Earth.

General Observers and Science Team members will use the full suite of JWST instruments to make dramatic progress in these and other areas at the forefront of astrophysics. In the next section, we highlight some of the programs the NIRCam team will be pursuing as illustrative of the contributions this instrument will be making.

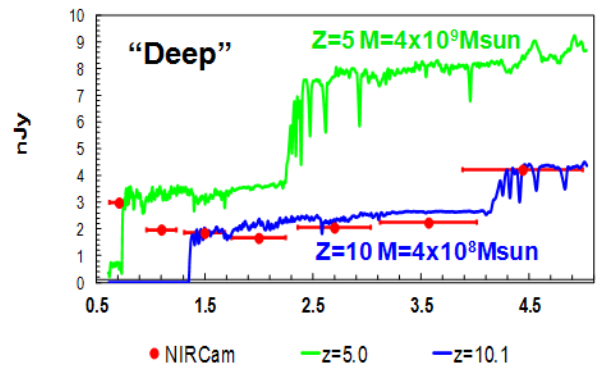

Figure 3. NIRCam will be able to detect distant galaxies out to $\mathrm{z} 10$ and estimate redshifts using photometry in multiple filters. To find such objects the NIRCam science team will conduct a number of "deep" multi-band surveys using integration times of 50,000 seconds/filter. 


\section{ILLUSTATIVE SCIENCE PROGRAMS}

\subsection{The End of the Dark Ages: First Light, Reionization, and Galaxy Formation}

The NIRCam guaranteed time extragalactic program focuses on the exploration of the epoch of reionization and the subsequent evolution of galaxies, including the roles of active galactic nuclei and environment. From the beginning, JWST has been designed to study galaxies at the redshift frontier, and its aperture and infrared wavelength coverage ensures that it will be the pre-eminent facility for this topic. Reionization around redshift 10 is the last major transformation of the baryonic content of the Universe, and we expect it to have significant implications for the formation of galaxies, as it is accompanied by a large increase in the temperature of the cosmic gas. The temporal and spatial pattern by which reionization occurs depends upon the ionizing sources and carries important ramifications for the small building blocks that will eventually form the mature galaxies that we see at later times.

Optical wavelengths are blind to the epoch of reionization due to absorption by the intergalactic medium of all light shortward of the Lyman-alpha transition of hydrogen (redshifted $\lambda_{\mathrm{o}}=121.6 \mathrm{~nm}$ ), thus making JWST's infrared reach critical. We will identify high-redshift galaxies in deep multi-band imaging using their distinctive colors, blue throughout the near-infrared before plummeting shortward of redshifted Lyman-alpha, and use JWST spectroscopy to confirm at least the brighter candidates (Figure 3). Deep (50-100 kilosec/band) images will reveal hundreds of objects per field at today's redshift frontier. The number, sizes, and clustering of the galaxies will all inform our studies of the causes and effects of cosmic reionization.

These same images, as well as shallower flanking fields, will be used to study the evolution of galaxies at less extreme redshifts. Here, JWST NIRCam's long-wavelength channel probes the rest-frame optical and does so with enough angular resolution to study the detailed morphology of the galaxies. JWST's NIRCam and NIRSpec can do at $z^{\sim 5}$ what HST imaging and ground spectroscopy have done at $z \sim 1-2$ (Figure 4). The list of topics is long: studying the evolution of star formation, stellar mass and metallicity buildup, morphological transformations, and galaxy activity, along with their correlations with environments and X-ray, far-IR, and radio properties.
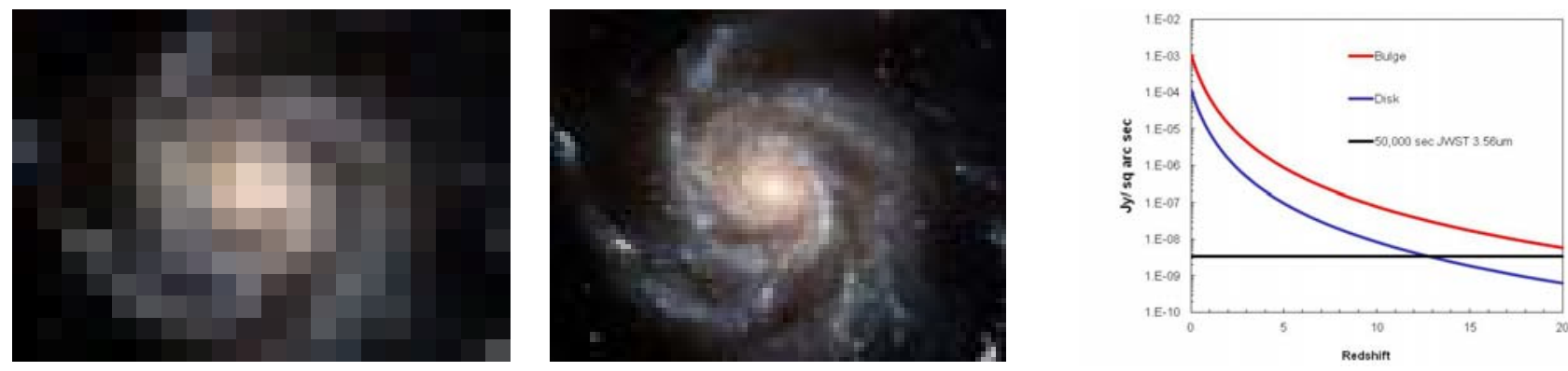

Figure 4. Simulation of a distant spiral galaxy as seen at the resolution of HST/WFC3 and b) as seen with JWST/NIRCam; c) the surface brightness sensitivity of NIRCam can follow the formation of galaxy bulges and disks out to $\mathrm{z} 10$.

\subsection{The Birth of Stars and Protoplanetary Systems}

Studying the formation and evolution of stars and planetary systems links the study of massive stars to the study of galaxy formation on the one hand (§2.1, above) while observations of debris disks around nearby stars is tied directly to the investigation of nearby planetary systems (§2.3, below). The key topics to be investigated by the NIRCam science team include the following:

What physical variables determine the shape of the IMF? The NIRCam team will make broad- and medium-band filter observations of distant clusters of stars covering a wide range $[\mathrm{Fe} / \mathrm{H}]$ to address the role of metallicity in the efficiency of star formation with obvious linkages to formation of stars in the early, metal-poor, Universe. 
How do cloud cores collapse to form isolated protostars? Extinction mapping against a background of distant stars will trace the internal density structure of molecular cloud cores. Narrow band imaging and spectroscopy will help to reveal the composition of grains and their evolution as a function of location within the cloud.

Does mass loss play a crucial role in regulating star formation? Narrow band imaging of embedded clusters will reveal the interaction between protostellar jets and outflows and prenatal environment.

What are the initial conditions for planet formation? Imaging of nearby young stars in medium and narrow bands with NIRCam's coronagraph will trace the structure and composition of disks during the dense protostellar phase (optical depth, $\tau>0.1$ ) when planets are presumed to form.

How do these disks evolve in structure and composition? ? The overall wavefront error of JWST limits NIRCam's ability to image debris disks to objects with optical depths $\tau>10^{-4}$ similar to the limiting performance of HST. However, NIRCam will be able to make compositional maps of ices in disks, because of its wavelength coverage, and to identify rings and other structures that might be linked to the presence of planets with angular resolution 2-3 times better than that of HST.

These questions and others will be addressed in conjunction with other JWST instruments, e.g. MIRI for debris disk mapping, and with other ground-based facilities such as ALMA for the structure of cloud cores.

\subsection{Planetary Systems and the Origins of Life}

\section{Direct Imaging of Planets}

While JWST does not have the large aperture or extreme wavefront control of some the large ground based telescopes equipped with Extreme Adaptive optics, e.g. the Gemini Planet Imager which is expected to achieve contrast ratios of $10^{-7}$ at $1 "$ at $1.6 \mu \mathrm{m}^{5}$, NIRCam is equipped with a variety of Lyot coronagraphs which will offer important gains in sensitivity at wavelengths where young planets are brightest, around 3-5 $\mu \mathrm{m}$. NIRCam's coronagraphs [2] will achieve stellar rejection of $\sim 10^{-4.5}$ at $1 "$ at $4.4 \mu \mathrm{m}$ (Figure $5 \mathrm{a}$ ) and is well suited to surveys of targets inaccessible to ground-based AO, namely faint stars such as late type M stars or distant young stars ${ }^{6}$. For such targets JWST NIRCam's coronagraph will allow detection of Saturn-mass planets at ages of $1 \mathrm{Gyr}$ or less (Figure 5b) located at a few 10s of AU from their host stars. The NIRCam team will characterize 10-15 previously known planetary systems, obtaining complete spectral
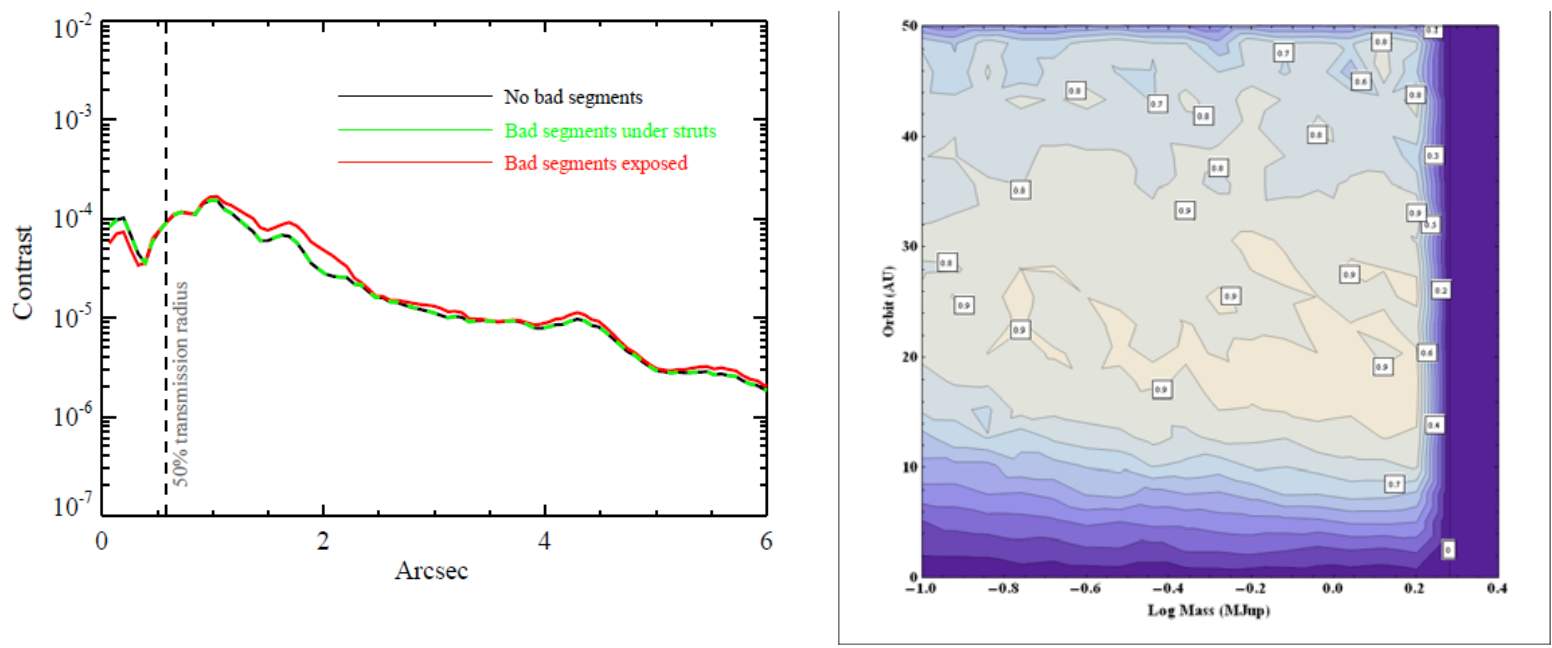

Figure 5 left, a) NIRCam's coronagraph will achieve starlight rejection of $10^{-4}$ to $10^{-5}$ outside of 1 " at $4.6 \mu \mathrm{m}$. The effects of two mirror segments of lower than average quality will be minimized by placing those segments behind the secondary mirror support struts which are masked out by the NIRCam Lyot mask. right, b) A Monte Carlo simulation ${ }^{6}$ of a coronagraphic survey at $4.4 \mu \mathrm{m}$ shows completeness contours $>80 \%$ for planets orbiting a sample of nearby M stars at separations between 20 and 50 AU and for masses greater than $0.1 \mathrm{M}_{\text {Jupiter }}$. 


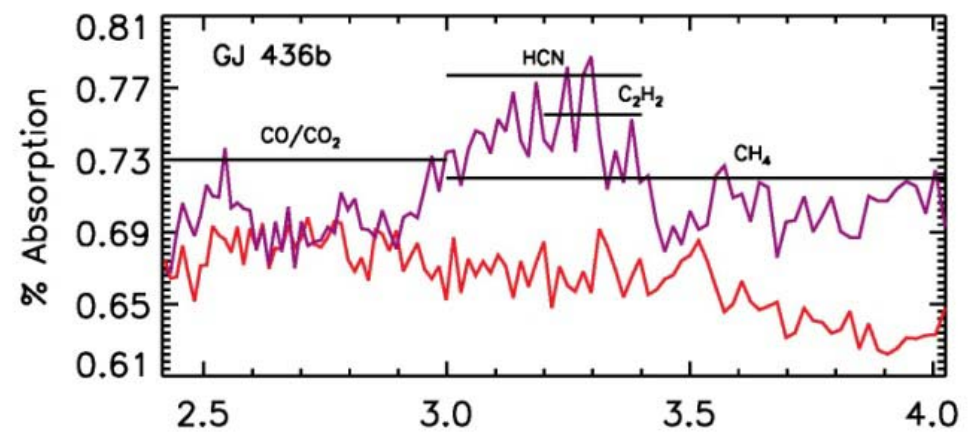

Figure 6. A simulated transit spectra of the exoplanet GJ 436b. The red curve is for a 30× solar composition with low water and methane abundance. The purple curve is for the same atmospheric abundances but with the addition of nonequilibrium photochemical hydrocarbon products generated by insolation from its host star ${ }^{9}$.

energy distributions (1-5 $\mu \mathrm{m}$ with NIRCam and 5-10 $\mu \mathrm{m}$ with MIRI) to compare with physical models, searching for additional lower mass planets in those systems, and refining orbital parameters. The team will also carry out a number of small surveys looking at faint $\mathrm{M}$ stars or systems where the spectral energy distribution suggests a ring-like disk indicative of the presence of planets. Another planet-related program will be the study of the closest, coldest, lowest mass brown dwarfs discovered with the WISE satellite. These Y dwarfs, a few of which are located within 10 pc of the Sun, have masses of 1-10 $\mathrm{M}_{\mathrm{Jup}}$ and ages of 1-10 $\mathrm{Gyr}^{7,8}$. The team will carry out a program of imaging to look for companions and to determine astrometric distances as well as spectroscopy to characterize their physical conditions.

\section{Transit Measurements}

The NIRCam grism is very well suited to acquiring diagnostic spectra of transiting and eclipsing exoplanets. Its 2.4 - 5 $\mu \mathrm{m}$ range is sensitive to numerous molecular features, and the 64 milli-arcsecond pixels of the long-wave camera allow for good spatial sampling of the spectrum with low systematic noise. Filters are used in series with the grism to select a subset of this spectral range. Figure 6 shows simulated transit spectra of the exoplanet GJ 436b with 2 different possible compositions ${ }^{9}$. The red curve is for a $30 \times$ solar composition with low water and methane abundance, as suspected for this planet. The purple curve is for the same atmospheric abundances with the addition of non-equilibrium photochemical hydrocarbon products generated by insolation from its host star. The simulation is for a single transit (1 hr total integration time) and includes the expected transmissions of JWST and NIRCam as well as photon noise and an expected systematic noise component of 50 parts per million ${ }^{10}$. This spectrum covers the range of the grism when used in series with the F322W2 filter. The simulated data were binned by a factor of 10 to a spectroscopic resolution of $\mathrm{R}=200$ to improve signal-to-noise. It is clear that a single grism observation can determine whether photochemistry is an important factor in the atmospheres of exoplanets. This information will allow significant advances in understanding the formation, compositions, chemistry, and dynamics of exoplanet atmospheres.

NIRCam will also make important photometric observations of transiting planets. JWST and NIRCam will be sensitive enough to confirm many of the small exoplanet candidate planets found by the Kepler mission to be in the habitable zones of their host stars. The large wavelength difference between Kepler (visible) and NIRCam ( $2 \mu \mathrm{m}$ region) will all but ensure that candidates producing equal amplitude absorptions in these bands are true transiting planets. JWST's targets for spectroscopic confirmation will consist primarily of planets orbiting stars brighter than $\mathrm{J}<7$ mag, of which fewer than 10 are presently known. It is only these brightest objects that offer enough stellar photons to permit extraction of high resolution planetary spectra. RV and transit surveys of very low mass M stars are presently underway to search for transiting planets. The TESS mission currently under consideration by NASA could produce dozens of transiting rocky planets orbiting bright stars in time for observation by JWST. The NIRCam team plans on expending 100-200 hours of guaranteed time to observe a small number of highly favorable transiting/eclipsing exoplanets in a program that will be coordinated with the other JWST instruments.

Transit measurements in general and spectroscopic measurements in particular demand highly precise, differential measurements at the level of $<50$ parts per million (ppm). Recent tests on H2RG detectors similar to those used in NIRCam indicate that residual detector errors can be reduced to a level of 10-20 ppm by a combination of careful 
calibration, averaging over multiple pixels and reduction of common mode error sources ${ }^{10}$. The ability to take grism spectra in the long wave length arm while tracking the pointing in the short wavelength arm offers a powerful ancillary dataset for removing the effects of pointing drifts on the photometry.

\subsection{Solar System Science}

Solar system science is an important component of the JWST mission. Because of their very high sensitivity, wavelength coverage, and spatial and spectral resolution, NIRCam and the other JWST instruments are particularly well-suited to the physical characterization of Kuiper Belt Objects (KBOs) which have orbits beyond that of Neptune and related objects such as comets and Centaurs which have orbits crossing those of the giant planets. Over the past 20 years, study of the dynamical interactions of KBOs with the giant planets has revolutionized our understanding of the dynamical history of the entire solar system, but relatively little is known about their sizes, albedos, masses, and composition. The largest objects (e.g. Pluto, Eris, Sedna) are known to have ices of $\mathrm{H}_{2} \mathrm{O}, \mathrm{CH}_{4}, \mathrm{~N}_{2}, \mathrm{CO}, \mathrm{CO}_{2}$, and $\mathrm{NH}_{3}$ on their surfaces based on 1 $-2.5 \mu \mathrm{m}$ spectra, but smaller objects are too faint for ground-based instruments. Constraints on the reflectance of KBOs from $2.5-5 \mu \mathrm{m}$ where strong fundamental absorption bands of these ices reside (Figure 7) are nearly non-existent, coming almost exclusively from the Spitzer IRAC instrument with its broad 3.6 and 4.5 $\mu$ m filters. NIRCam, with its $10 \%$ bandpass filters, could detect KBOs as small as $200 \mathrm{~km}$ diameter at signal to noise ratios of at least 20 across the entire $1-5 \mu \mathrm{m}$ band and thus allow for a sensitive search for molecular ices on the surfaces of a large sample of KBOs. Objects with particularly interesting colors could be followed up with NIRSpec or the NIRCam grism. The spatial resolution and sensitivity of NIRCam will permit accurate astrometry to determine orbits and masses of KBO binaries. NIRCam will also be able to measure the colors and surface properties of comet nuclei as small as $1 \mathrm{~km}$ when they are as far as 6 AU from the Sun, thereby avoiding contamination by the coma. By using subarrays, NIRCam will also be able to image all of the giant planets through many or all of its filters (Figure 8), and could even image Mars in a few filters.

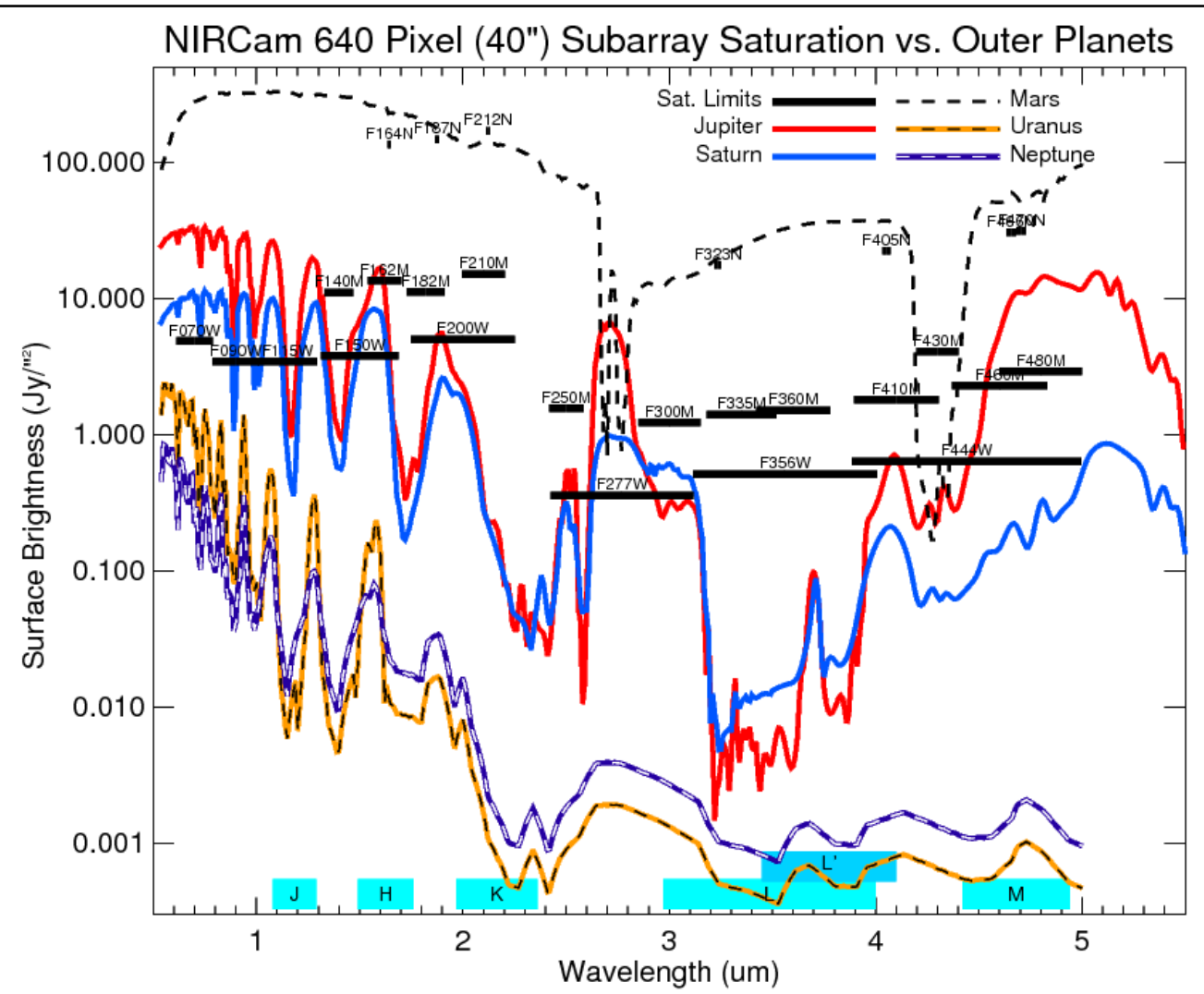

Figure 7. Saturation limits in various filters (black lines), are compared to spectra for the 5 outer planets (see legend). Despite their brightness, NIRCam will be able to make images of Jupiter, Saturn, Uranus and Neptune in all or many of its filters, and even Mars could be observed in a few filters. 


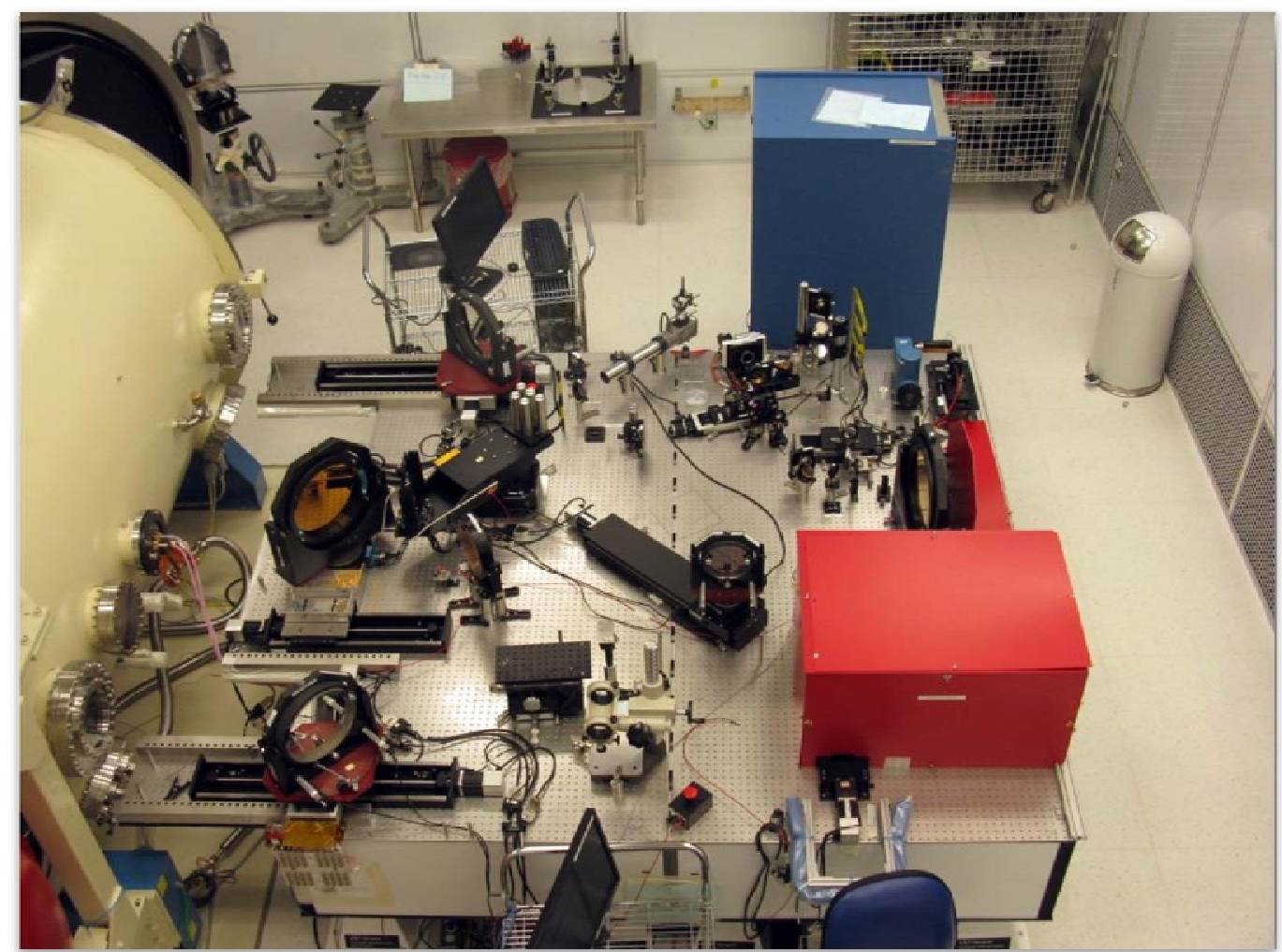

Figure 8.The Optical Metrology Assembly (OMA) which serves as a telescope simulator for testing NIRCam's modules. The chamber on the left is the cryogenic chamber which can hold both of NIRCam's modules at once.

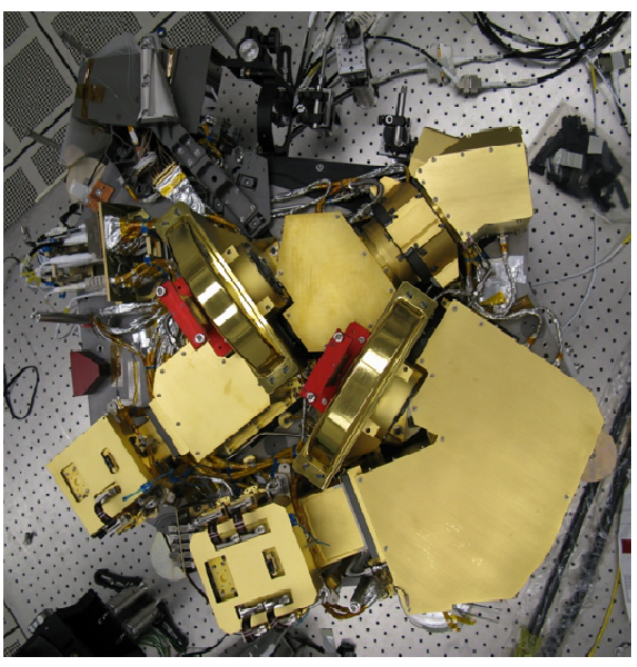

Module A

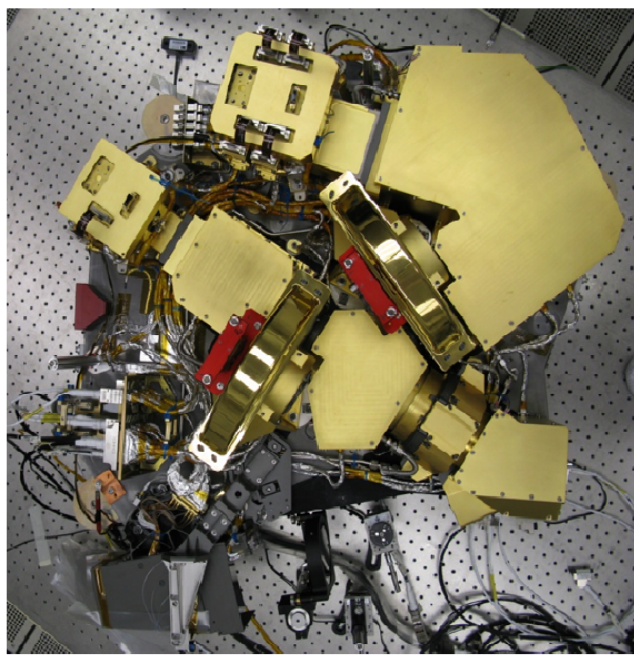

Module B

Figure 9. Fully assembled NIRCam modules on the assembly table. The red metrology reference fixtures will be removed before test. 


\section{NIRCAM STATUS AND TECHNICAL CHALLENGES}

\subsection{Current Status: Integration and Test}

The entire NIRCam instrument is now entering its cryogenic test cycle at Lockheed Martin before delivery to the JWST project at Goddard Space Flight Center (GSFC) for integration into the Integrated Science Instrument Module (ISIM). The test program at Lockheed Martin will encompass a broad range of tests including: detector performance (dark current, read noise, latent images), focus determination, pupil imaging and alignment, filter wheel alignment and repeatability, plate scale and optical distortion determination, image and wave front quality over the full field using phase retrieval at multiple field points, validation of wave front error models, wavelength calibration of the long wavelength grisms, plus a variety of tests related to NIRCam's role in wavefront sensing for the overall JWST optical system. Delivery of NIRCam to GSFC is expected later this year.

\subsection{Current Status: Performance}

All subsystem tests of all components of NIRCam have been completed and indicate that the sensitivity goals of NIRCam will be achieved across the 1-5 $\mu \mathrm{m}$ band. In particular, the quantum efficiency ( $80-90 \%$ at $2 \mu \mathrm{m}$ ), Correlated Double Sample read noise (10-20 $\left.\mathrm{e}^{-}\right)$and dark current $\left(<0.001 \mathrm{e}^{-} \mathrm{s}^{-1}\right.$ at $\left.39.5 \mathrm{~K}\right)$ of the H2RG detectors as well as the transmission of NIRCam's optical elements meet their specifications with the result that the magnitude limits presented in Table 1 and described elsewhere ${ }^{1}$ in more detail remain realistic estimates of NIRCam's performance. Table 2 gives sensitivities applicable to deep surveys in NIRCam's broad filters using as-built data.

Table 2. Sensitivity applicable to deep surveys using as-built data.

\begin{tabular}{|l|c|l|c|}
\hline Filter & $\begin{array}{c}\text { 5-sigma in 50,000 } \\
\text { seconds (nJy) }\end{array}$ & \multicolumn{1}{|c|}{ Filter } & $\begin{array}{c}\text { 5-sigma in 50,000 } \\
\text { seconds (nJy) }\end{array}$ \\
\hline F070W & 4.7 & F200W & 2.0 \\
\hline F090W & 3.3 & F277W & 2.9 \\
\hline F115W & 3.0 & F356W & 2.6 \\
\hline F150W & 2.5 & F444W & 4.9 \\
\hline
\end{tabular}

New detectors are now being fabricated at Teledyne with an additional barrier layer between the gold contact and the Indium bump bond on each pixel to address an ageing problem that has resulted in a sharp increase in the number of "hot pixels" in current generation of JWST's H2RG long wavelength detectors. Initial tests of the new batch of detectors indicate that key performance specifications meet NIRCam's requirements (Table 3). Once fabrication and testing of the new short and long wavelength detectors is complete in 2013, these new detectors will be retrofitted at GSFC into NIRCam.

Table 3. Comparison of existing H2RG detectors with newly fabricated devices.

\begin{tabular}{|c|c|c|c|}
\hline Property & Requirement & $\begin{array}{c}\text { Results from One } \\
\text { New Process Short- } \\
\text { wavelength } \\
\text { Detector }\end{array}$ & $\begin{array}{c}\text { Range for 8 Old Process } \\
\text { Short-wavelength Flight } \\
\text { Detectors }\end{array}$ \\
\hline Dark Current at 39.5K (e-/sec) & $<0.010$ & 0.008 & $0.0018-0.009$ \\
\hline Correlated Double Sample Read Noise (e-) & $<21$ & 20.3 & $15.9-20.5$ \\
\hline Total Noise in 1600 sec (e-) & $<9.0$ & 7.6 & $4.9-6.7$ \\
\hline Well Depth (e-) & $>60,000$ & 104,000 & $98,300-107,000$ \\
\hline Quantum Efficiency at $2 \mu \mathrm{m}$ & $>0.80$ & 0.81 & $0.80-0.99$ \\
\hline
\end{tabular}


Another critical performance requirement of NIRCam concerns its optical quality. This aspect of NIRCam is of particular importance because NIRCam serves to sense and provide wave front error for JWST as a whole, including all the other instruments. The NIRCam requirement for wave front error (WFE) is $69 \mathrm{~nm}$ over at least $80 \%$ of the FOV and no field point worse than $90 \mathrm{~nm}$. Careful analysis combined with a comparison with warm and cold optical tests have demonstrated that NIRCam will have $69 \mathrm{~nm}$ or less WFE over the entire FOV.

\section{EDUCATION AND PUBLIC OUTREACH}

The NIRCam team conducts an active program in education and public outreach (E/PO). In partnership with the Girl Scouts of the USA, we host "Train the Trainer" workshops for adult leaders from all Girl Scout Councils and also work directly with students and leaders in minority communities throughout southern Arizona and near Rochester, NY. Informal educators, like the GSUSA, help maintain long-term student interest in science, technology, engineering, and math (STEM) and strengthen classroom performance, building confidence and expertise. Together we foster girls' interest and creativity in these subjects, creating an environment that encourages such interests early in life.

Since 2002, our team has led twenty "Train the Trainer" workshops involving 225 adult leaders (41 U.S. states, Guam, Japan) in "holistic" STEM education combined with mission-specific concepts relating to the James Webb Space Telescope (JWST). We train the leaders to understand JWST's science themes. However, each theme involves several levels of abstraction and requires a strong foundation in basic astronomy. Through online networking we continue to help these leaders grow in the process of scientific inquiry and to equip them to host astronomy-related STEM activities at the troop level. Our workshops helped revise the national GSUSA badge curriculum and directly benefitted thousands of young girls of all ages.

Graduate students have contributed greatly to our overall strategic approach, leading to an emphasis on the learning process. They have developed and led activities in basic astronomy (night sky, phases of the Moon, the scale of the Solar System and beyond, stars, galaxies, telescopes, etc.) as well as JWST-specific research areas in extrasolar planetary systems and cosmology, to pave the way for girls and women to understand the first images from JWST. To date, twelve astronomy graduate students from the University of Arizona and UC-Santa Cruz have become active leaders in NIRCam's E/PO programs. The resulting experience has empowered these students to propose and to develop their own $\mathrm{E} / \mathrm{PO}$ programs after graduation as postdocs and young faculty.

\section{CONCLUSION}

The NIRCam instrument is on track to be delivered to the project later this year. NIRCam will satisfy all its fundamental requirements as to sensitivity, wave front quality, and with a breadth of capabilities encompassing wide field imaging in multiple filters, long wavelength grism spectroscopy, and coronagraphy. The NIRCam science team will exploit these capabilities in targeted studies in a few key areas while the broad community will use NIRCam to push the boundaries of astrophysics across many fronts, some known today and some not yet imagined.

\section{ACKNOWLEDGEMENTS}

The research described in this publication was carried out in part at the Jet Propulsion Laboratory, California Institute of Technology, under a contract with the National Aeronautics and Space Administration. Support from Near-IR Camera (NIRCam) instrument on James Webb Space Telescope via contract NAS5-02105 is gratefully acknowledged. Copyright 2011 by California Institute of Technology.

\section{REFERENCES}

[1] Rieke, M. J., Kelly, D. and Hoerner, S. "Overview of James Webb Space Telescope and NIRCam's Role", Proc. SPIE , 5904-01 (2005) 
[2] Krist, J. E. et al. "Hunting Planets And Observing Disks With The JWST NIRCam Coronagraph", Proc. SPIE, 6693-01 (2007).

[3] Greene, T. et al., "Observing exoplanets With The JWST NIRCam Grisms", Proc. SPIE, 669315 (2007).

[4] Gardner, J. P. et al. "The James Webb Space Telescope", Space Science Reviews, 123, 485 (2006).

[5] Macintosh, B.A. et al. 2008, Proc. SPIE, 7105-31.

[6] Beichman, C. et al., "Imaging Young Giant Planets From Ground and Space", PASP, 122,162 (2010).

[7] Cushing, M. et al., "The Discovery of Y Dwarfs using Data from the Wide-field Infrared Survey Explorer (WISE)" , Ap.J., 743,50 (2011).

[8] Beichman, C. et al. , "The Coldest Brown Dwarf (Or Free Floating Planet)?: The Y Dwarf Wise 1828+2650", Ap.J. submitted (2012)

[9] Shabram, M., Fortney, J. J., Greene, T. P., Freedman, R. S., "Transmission Spectra of Transiting Planet Atmospheres: Model Validation and Simulations of the Hot Neptune GJ 436b for the James Webb Space Telescope", Ap.J. 727, 65 (2011).

[10] Clanton, C. , Beichman, C., Vasisht, G. Gaudi, B.G., Smith R., "Precision Near Infrared Photometry For Exoplanet Transit Observations - I : Ensemble Spot Photometry for An All-Sky Survey", PASP, 124, in press (2012) 